\title{
Les notables et les forces politiques de droite en Bretagne dans les années 1930
}

\section{Christian Bougeard}

\section{(C) OpenEdition \\ 1 Journals}

\section{Édition électronique}

URL : http://journals.openedition.org/abpo/1564

DOI : $10.4000 /$ abpo. 1564

ISBN : 978-2-7535-1487-4

ISSN : $2108-6443$

Éditeur

Presses universitaires de Rennes

Édition imprimée

Date de publication : 20 septembre 2002

Pagination : 121-139

ISBN : 978-2-86847-768-2

ISSN : 0399-0826

\section{Référence électronique}

Christian Bougeard, «Les notables et les forces politiques de droite en Bretagne dans les années 1930 », Annales de Bretagne et des Pays de l'Ouest [En ligne], 109-3 | 2002, mis en ligne le 20 septembre 2004, consulté le 01 mai 2019. URL : http://journals.openedition.org/abpo/1564 ; DOI : 10.4000/ abpo. 1564 


\title{
Les notables et les forces politiques de droite en Bretagne dans les années 1930
}

\author{
Christian BOUGEARD \\ professeur d'histoire contemporaine \\ CRBC-UMR 6038 du CNRS, Université de Bretagne Occidentale - Brest
}

En mai 1936, les électeurs bretons se prononcent majoritairement pour des candidats hostiles au Front populaire confortant l'image d'une région catholique et conservatrice solidement ancrée à droite. En effet, dans les cinq départements bretons, trente députés de droite et du centre s'imposent contre quatorze députés seulement soutenant le Front populaire (six radicaux-socialistes et huit $\mathrm{SFIO})^{1}$. Mais la réalité politique est plus complexe. Dans une région de tradition politique modérée, les effets économiques et sociaux de la Grande Dépression et les enjeux électoraux de 1936 exacerbent les contradictions et l'affrontement politique en deux blocs antagonistes. Le mécontentement des campagnes qui monte de 1932 à 1935 bouscule les notables qui encadrent la vie politique depuis l'avant Première Guerre mondiale ou depuis 1919, à gauche comme à droite. Imitant les partis de gauche (SFIO et PCF et secondairement les radicaux-socialistes), les notables de droite qui s'appuyaient dans les années 1920 sur " des structures électorales éphémères ${ }^{2}$ " tentent d'organiser des partis politiques dans les années 1930. Ils cherchent aussi à canaliser ou à récupérer le mécontentement qui s'exprime dans les campagnes par le biais du dorgérisme, de 1930 à 1936 mais aussi après 1936 contre le Front populaire. À côté des anciens notables, solidement enracinés dans leur circonscription, des hommes nouveaux qui émergent vont s'appuyer sur des partis plus offensifs pour faire pièce à la fois au militantisme de gauche et à la montée en puissance du parti social français (PSF) du colonel de La

1. Nantes et Saint-Nazaire élisent quatre socialistes tandis que les campagnes blanches envoient cinq députés de droite (deux Fédération républicaine et trois nobles apparentés au Palais-Bourbon). Ces trois aristocrates sont les héritiers directs du traditionalisme et de l'Action française. La situation des droites de Loire-Inférieure mériterait une étude spécifique.

2. SAINCLIVIER, Jacqueline, L'Ille-et-Vilaine 1918-1958. Vie politique et sociale, Rennes, PUR, 1996, p. 77-79. 
Rocque. Après avoir dressé un tableau de la droite bretonne dans les années 1930, il convient de s'interroger sur les évolutions de 1936 à la guerre et sur les éventuels reclassements des hommes et les passerelles entre les forces de droite.

\section{Structuration des forces de droite dans les années 1930-1934}

Très sensible à la question religieuse et à l'affrontement de l'école laïque et de l'école privée, clivage politique majeur entre bleus et rouges d'un côté, blancs de l'autre, l'électorat de droite breton se sent menacé lorsque la gauche parvient au pouvoir comme avec le Cartel des gauches en 1924 ou la victoire des gauches en $1932^{3}$. Dès lors, à l'appel de la Fédération nationale catholique (FNC) et de la hiérarchie de l'Église, tous les réseaux catholiques et toutes les droites se mobilisent massivement contre les gouvernements comme lors des grands rassemblements dans la France de l'Ouest en 1925 et $1926^{4}$. Le quotidien régional L'Ouest-Éclair, fondé par les abbés démocrates en 1899 dont l'abbé Trochu, appuie cette mobilisation des catholiques et contribue à l'émergence d'une nouvelle force politique qui va compter et bousculer la droite bretonne, celle des démocrates populaires du PDP ${ }^{5}$. Contre la droite conservatrice, ces héritiers du Sillon, fermes défenseurs de la République, ont conquis des positions électorales dans le Léon, en Ille-et-Vilaine et dans le Morbihan.

Au début des années 1930, la question sociale et les conséquences de la grande dépression tendent à déplacer la polarisation politique contre les majorités gouvernementales impuissantes à réagir. Ainsi, aux élections législatives de 1932, le balancier électoral en Bretagne comme en France repart vers la gauche au profit du parti radical-socialiste. Au $1^{\text {er }}$ tour, avec $32,2 \%$ des inscrits, les gauches (en net progrès dans le Morbihan) devancent les droites, 30,2\%, qui se replient sur leurs bastions du Léon et des marges orientales avec un recul de $5 \%{ }^{6}$. Avec $17,4 \%$, les centres progressent. En Bretagne, il s'agit généralement d'hommes attachés à la laïcité considérés comme des modérés de centre gauche ou de centre droit ${ }^{7}$. Au second tour, les radicaux-socialistes (dix élus) et la SFIO (cinq députés) gagnent six sièges et de nombreux centristes républicains évoluant à la lisière du centre gauche et du centre droit sont réélus avec des voix de gau-

3. Bougeard, Christian, La Bretagne d'une guerre à l'autre 1914-1945, Paris, éd. JeanPaul Gisserot, 1999.

4. BEnsoussan, David, «Le réveil des catholiques bretons (1924-1926) ", Vingtième siècle, $\mathrm{n}^{\circ} 57$, janvier-mars 1998 , p. 57-75

5. Voir chapitre 6 de Jean-Claude DeLBREIL, L'Ouest-Éclair. Naissance et essor d'un grand quotidien régional, LaGReE, Michel, HARISMENDY, Patrick et Denis, Michel (dir.), Rennes, PUR, 2000.

6. PIERRE, Patrick, Les Bretons et la République. La construction de l'identité bretonne sous la Troisième République, Rennes, PUR, 2001, p. 181.

7. BOUGEARD, Christian, «Itinéraires de parlementaires modérés en Bretagne des années 1920 aux années 1960 ", Les modérés dans la vie politique française (1870-1965), Roth, François (dir.), Nancy, PUN, 2000, p. 209-223. 
che (une douzaine) ${ }^{8}$. Le pays gallo, en Haute Bretagne, fournit le gros des troupes des douze députés de droite (dont quatre indépendants, quatre Fédération républicaine-URD, trois centre républicain) alors que le PDP conserve cinq députés ${ }^{9}$.

C'est durant cette période du début des années 1930 que, dans certains départements, des partis de droite s'efforcent de s'organiser alors que plusieurs grands notables gagnent le Sénat tout en évoluant du centre droit vers la droite. C'est le cas par exemple du polytechnicien poincariste Yves Le Trocquer, élu en 1919 dans les Côtes-du-Nord sur la liste d'Union républicaine avec l'étiquette républicain de gauche contre une liste de droite ${ }^{10}$. Ministre des Travaux publics de 1920 à 1924, leader du Bloc national en 1924, Le Trocquer préfère gagner le Sénat en octobre 1929, laissant son siège à Oswen de Kéroüartz (député de Guingamp-2 de 1930 à 1936). De Kéroüartz porte la même étiquette de républicain de gauche mais il est le fils d'un grand propriétaire terrien, ancien député (1898-1902) et sénateur (1912-1919) de droite des Côtes-du-Nord ${ }^{11}$. L'itinéraire politique d'Yves le Trocquer illustre parfaitement le glissement vers la droite dans les années 1930 de certains notables laïques contestés par une gauche radicale socialiste ou socialiste SFIO de plus en plus combative. Au Sénat, il est élu président du groupe de l'union démocratique et radicale et il devient vice-président du parti de l'Alliance républicaine démocratique (ARD) ${ }^{12}$. Dans sa région, il nous semble évoluer nettement à droite. Mais il ne peut être assimilé à la droite catholique même s'il a recherché une entente avec les démocrates populaires pour une liste commune en 1924. Son collègue Maurice Bouilloux-Lafont, député de Quimper depuis 1914, membre de l'ARD, puis du parti républicain démocratique et social (PRSD) de 1920 à 1926, a suivi le même parcours jusqu'à sa défaite en 1932 face au radical-socialiste (RS) Pierre Pouchus ${ }^{13}$. Lui aussi avait tenté une entente avec les démocrates chrétiens en 1919, rejetée aussi bien par les radicaux socialistes intransigeants comme Georges Le Bail que par les catholiques conservateurs. Ces deux exemples illustrent bien l'impossible convergence au centre, en

8. En 1932, ils se répartissent dans les groupes indépendants de gauche, républicains de gauche et gauche radicale. Pour les biographies des députés voir PASCAL, Jean, Les députés bretons de 1789 à 1983, Paris, PUF, 1993.

9. Paul Trémintin (1924) est réélu dans le Léon avec Paul Simon.

10. La liste d'union républicaine compte des hommes de gauche (deux républicains socialistes et un radical socialiste), des hommes de centre gauche et de centre droit.

11. BOUgEARD, Christian, Le choc de la guerre dans un département breton : les Côtes-duNord des années 1920 aux années 1950, thèse d'État, Rennes 2, 1986, p. 93-95 et 175-178. Pour le Sénat, Le Trocquer a tenté de se faire investir par " le congrès républicain " de plus en plus orienté à gauche et a été battu. Il est finalement élu au $3^{\text {e }}$ tour sénateur contre un radical socialiste nettement de gauche grâce à des voix de droite. De Kéroüartz est réélu en 1932 avec l'étiquette URD, seul candidat député à droite des Côtes-du-Nord.

12. SANSON, Rosemonde, «L'Alliance républicaine démocratique : une reformulation du Centre gauche?", Les modérés dans la vie politique française, op. cit., p. 155-167.

13. CARO, Laurent, Le Finistère et Maurice Bouilloux-Lafont député de Quimper (19141932). Ligne politique (1914-1939), maîtrise d'histoire (M. H.), Brest, Université de Bretagne Occidentale (UBO), 1996. 
Bretagne, de républicains laïques et catholiques dans les années 1920, ce qui a rejeté à droite électoralement les hommes du PDP. Cependant, opposés aux gouvernements de gauche de 1932 à 1934 et surtout au Front populaire après 1936, ces notables centristes et laïques évoluent vers la droite comme leur parti l'Alliance républicaine démocratique.

Ces leaders ont assuré la représentation départementale de l'ARD sans qu'on puisse toujours en saisir une organisation permanente en dehors des périodes électorales. L'activité du parti tient beaucoup à la présence d'élus sur le terrain. Pourtant, à la fin de 1933 et au début de 1934, après la prise de direction par Pierre-Étienne Flandin, l'ARD semble s'organiser dans la région à la suite de tournées de propagande. Dans les Côtes-du-Nord, R. Jossier, membre du comité directeur, invité par l'ancien député (19241928) et maire de Saint-Brieuc (1919-1925) Armand Waron, attire peu de monde dans ses réunions ${ }^{14}$. Dénonçant " la gabegie actuelle " des gouvernements de gauche, les scandales politico-financiers et une fiscalité inadaptée, il appelle à la réforme de l'État, un thème à la mode repris par toutes les droites notamment par André Tardieu, le vaincu de 1932. Il s'agit, surtout après le choc du 6 février, d'organiser une fédération et de soutenir le gouvernement de Gaston Doumergue auquel participent les dirigeants issus de l'ARD, André Tardieu et Louis Barthou. Au début de 1935, ce parti compterait environ 200 membres dans les Côtes-du-Nord dont 30 à Saint-Brieuc, de recrutement urbain, principalement dans la bourgeoisie commerçante ${ }^{15}$. À l'approche des élections municipales de 1935, et contre le Front populaire, le glissement à droite est très net. Des responsables de l'ARD participent à " un comité républicain anticollectiviste " à Saint-Brieuc et en animent un à Dinan ${ }^{16}$. À Lannion, au début 1935, des papillons de ce comité dénoncent « les buts du Front populaire : violer, tuer, incendier ». Des notables républicains modérés s'alignent désormais sur une droite dure reprenant le discours et les thématiques du Centre de propagande des républicains nationaux qui mène des campagnes d'affiches. Mais l'Alliance qui n'a plus les moyens de présenter un candidat aux élections cantonales à SaintBrieuc, à la fin 1934, semble divisée entre une aile droite et une aile gauche qui serait prête à soutenir un candidat radical indépendant. Appuyée principalement sur des élus locaux portant l'étiquette des républicains de gauche, l'ARD voit son influence décliner de 1936 à 1939. Après la mort du sénateur Le Trocquer le 21 février 1938, on ne trouve plus trace d'une organisation militante dans les Côtes-du-Nord.

Dans les milieux catholiques, des militants issus du Sillon vont former des fédérations du PDP en Bretagne à partir de 1925. La Fédération des républicains démocrates du Finistère (FRDF) a joué un rôle pionnier en s'organisant de 1911 à 1913 pour soutenir l'avocat Paul Simon, élu député

14. Bougeard, Christian, thèse citee, p. 360-362.

15. Il est dirigé par deux représentants de commerce dont J. Waron, le fils de l'ancien maire.

16. C'est le cas de l'industriel Busnel, conseiller d'arrondissement, thèse citée p. 372-373. 
dans le Léon (Brest-2) dès 1913 contre un conservateur ${ }^{17}$. Ces catholiques républicains vont tenter dans l'entre-deux-guerres de se situer au centre de l'échiquier politique en s'opposant électoralement à la droite la plus conservatrice, héritière de la tradition monarchiste souvent légitimiste. Mais en Bretagne, ils sont perçus par leur électorat et leurs adversaires de gauche comme appartenant au camp des droites. Et de fait, face à la gauche radicale et socialiste (élections de 1924, politique du Cartel des gauches, 1932-1934, Front populaire), ils se retrouvent le plus souvent aux côtés de la droite conservatrice tout en plaidant la concentration des républicains du centre ${ }^{18}$. Organisé au niveau national en 1925, le PDP peut compter en Bretagne sur le soutien et le financement du quotidien rennais L'Ouest-Éclair, notamment de ses fédérations ${ }^{19}$. Emmanuel Desgrées du Loû, autre fondateur du journal, est vice-président national du PDP et président de la fédération d'Ille-et-Vilaine créée le 15 mars $1925^{20}$. À sa mort en 1933, le député finistérien Paul Simon, vice-président de la fédération finistérienne et du parti, lui succède comme directeur politique du journal, ce qui traduit les liens étroits entre le PDP et le quotidien régional catholique républicain qui s'est affronté à l'Action française et à la droite la plus conservatrice dans les années $1920^{21}$.

Une fédération s'organise dans le Morbihan, présidée par le journaliste Ernest Pezet, collaborateur de Marc Sangnier avant 1914, cofondateur en 1919 de l'Union nationale des combattants (UNC), puis du PDP. En 1928, E. Pezet a été élu député de Vannes-2 contre le monarchiste Georges de Chabannes. En 1932, il est réélu dès le $1^{\text {er }}$ tour avec $74 \%$ des voix contre un candidat de l'URD qui n'a recueilli que 19,6\% ${ }^{22}$. Au tournant des années 1930, dans ces régions blanches du Léon, du Vannetais, voire du Vitréen, où les gauches sont très faibles, l'affrontement électoral a lieu au sein des droites entre des notables héritiers de la tradition conservatrice et des démocrates populaires défendant la République en position centriste. Le

17. FLoch, Jean-Charles, Les républicains démocrates du Finistère (1911-1940), M. H., Brest, UBO, 1990.

18. Cinq des six députés du Finistère élus en 1919 sur la liste de droite siègent au groupe des républicains de gauche avec Le Trocquer. Trois sont des cadres et des élus de la FRDF du Finistère (Paul Simon, Victor Balanant, battu à Quimperlé en 1928 et Jean Jadé battu à Quimper II en 1932). Avec la création du PDP, cette convergence avec des républicains laïques centristes de l'ARD échoue. Dans le Finistère sud, les PDP sortants sont battus par des radicaux socialistes clairement de gauche, ce qui positionne les démocrates chrétiens dans le camp de la droite.

19. DELBREIL, Jean-Claude, Centrisme et démocratie-chrétienne en France. Le PDP des origines au MRP (1919-1944), Publications de la Sorbonne, 1990. Par exemple, L'Ouest-Éclair imprime gratuitement jusqu'en 1932, Le Petit Démocrate, le journal national du parti. Voir DELBREIL, op. cit.

20. SAINCLIVIER, Jacqueline, op. cit., p. 85-88.

21. Le PDP d'Ille-et-Vilaine a un élu à Rennes-Montfort, Étienne Pinault, député de 1906 à 1910 et de 1928 à 1940 .

22. DUFRESNE, Martine, Les campagnes électorales des élections législatives de 1932 à 1936 dans le Morbihan, M. H., Rennes 2, 1996, p. 96-100. Notons que les trois candidats de gauche (radical indépendant, SFIO, PCF) se sont partagé $6,2 \%$ des voix... 
bloc des droites vole en éclat. L'Église catholique ne prend plus position. Le soutien de L'Ouest-Éclair et des réseaux catholiques et agricoles fait la différence. Comme Paul Simon ou Pierre Trémintin, président de la fédération du Finistère, Ernest Pezet joue un rôle important dans l'animation des fédérations bretonnes du PDP. Le 12 avril 1934, il participe au congrès de celle des Côtes-du-Nord à Tréguier qui compte environ 500 adhérents dont l'ancien député de Lannion Yves Le Cozannet (1930-1932) qui semble y avoir adhéré ${ }^{23}$.

Plus à droite, dans les années 1920 la Fédération républicaine voit son implantation dans les régions blanches limitée par l'enracinement, souvent dynastique car nobiliaire, de grands notables conservateurs dans l'est de la Bretagne notamment, avec les marquis députés de Loire-Inférieure, mais aussi dans les conseils généraux et dans les communes. En 1932, son influence à la Chambre se réduit car la Fédération républicaine perd deux sièges de député : Victor Le Guen, député de Saint-Brieuc-2 depuis 1921 et Joseph Cadic député de Pontivy-1 depuis 1924, réélu en 1928 avec le soutien de L'Ouest-Éclair ${ }^{24}$. Du fait d'une scission, l'URD ne conserve que quatre inscrits bretons ${ }^{25}$. Mais ce sont souvent des personnalités influentes comme Alexandre Lefas, député d'Ille-et-Vilaine depuis 1902, président du conseil général de 1925 à 1928, qui rejoint le Sénat, ou le $\mathrm{D}^{\mathrm{r}}$ Louis Guillois, maire (1902) et conseiller général de Ploërmel, sénateur du Morbihan de 1920 à 1932 puis député de 1932 à 1936. Dans sa profession de foi de 1932, il se veut le porte-parole des républicains nationaux et s'affirme comme " un catholique convaincu ", défenseur de l'école privée, de la famille (nombreuse) et des anciens combattants ${ }^{26}$. Il revendique aussi ses responsabilités au sein de la Fédération républicaine comme membre du conseil national et comme président de la section départementale, preuve d'une organisation structurée dans le Morbihan.

Dans les Côtes-du-Nord, la Fédération républicaine s'organise d'abord dans la région de Dinan pour lutter contre Michel Geistdoerfer, le nouveau député-maire (1928-1929), un radical-socialiste nettement de gauche. L'ancien maire de la cité, H. Durand forme le 8 février 1930 un comité d'arrondissement assisté du conservateur de Pontbriand. Avec plusieurs maires du département, le 9 mars, il accueille Louis Marin qui expose le programme du parti devant 1800 personnes dont de nombreux paysans. Le 30 avril 1933, le vice-président Georges Bonnefous déplace encore un millier

23. Le parti revendique plusieurs maires et lance en juin 1934 un journal, Le Démocrate des Côtes-du-Nord.

24. La liste de la Fédération républicaine indépendante a obtenu 5 sièges sur 8 dans le Morbihan en 1924.

25. Il s'agit de Vincent Inizan, député du Finistère (Brest-3) de 1919 à 1940 et de De Montaigu, député de Saint-Nazaire-2 de 1910 à 1919 et de 1928 à 1940. Trois députés bretons s'inscrivent au groupe du Centre républicain d'André Tardieu issu de l'URD, le groupe parlementaire de la Fédération républicaine : Bret (Redon, 1924-1940), de Lyrot (Vitré, 1932-1940) et Merland (Nantes-1, 1924-1936).

26. DUFRESNE, Martine, op. cit., annexe 1. 
de personnes. La capacité de mobilisation de la Fédération républicaine semble assez forte sans qu'il y ait vraiment d'organisation ni de vie militante suivie. Elle s'apparente à l'Ille-et-Vilaine voisine qui ne dispose que de comités électoraux plus ou moins éphémères ${ }^{27}$. Mais précisément, à partir de 1933, la capacité de mobilisation des droites s'appuie sur des méthodes et des moyens qui dépassent une organisation partisane souvent légère.

\section{Les droites bretonnes dans les affrontements de 1933 à 1936}

Région modérée dans la diversité de ses "pays ", où les catholiques se sont ralliés à la République au début des années 1890, la Bretagne n'est guère concernée par les mouvements et les ligues d'extrême droite, surtout après la condamnation de l'Action française (AF) par Pie XI en décembre 1926. Quelques nobles restent fidèles à l'AF dans les Côtes-du-Nord mais les rares sections urbaines (Dinan, Saint-Brieuc, Lannion) n'ont plus les moyens de tenir que de rares réunions privées (en 1928-1929 et en 1935). La situation est comparable en Ille-et-Vilaine ${ }^{28}$. En Loire-Inférieure surtout, dans le Morbihan et dans le Finistère (une fédération de 300 membres en 1928), des propriétaires fonciers ou des officiers catholiques, souvent nobles, restent de sensibilité Action française en s'appuyant sur les mairies qu'ils conservent. Un seul grand rassemblement militant est signalé à Saint-Goazec (Finistère) en 1933 : Léon Daudet déplace encore 2000 à 3000 personnes $^{29}$.

Certains vont ensuite militer dans les ligues antiparlementaires et leur fournir des cadres qui vont rejoindre le PSF. Mais jusqu'en 1933 ou 1934, les ligues n'ont guère d'existence dans la région hormis quelques réunions à Rennes ou à Nantes qui n'attirent pas grand monde. La Loire-Inférieure constitue un cas à part car c'est le seul département de l'Ouest qui compte plus de 10 sections des Jeunesses Patriotes (JP) en $1932^{30}$. À Saint-Brieuc, le 22 juin 1935, on observe la convergence de militants de l'AF et des JP. Pourtant ces JP ne concernent que quelques dizaines de jeunes, souvent des étudiants à Rennes, recrutant très peu d'adhérents dans les Côtes-duNord (Saint-Brieuc et Paimpol). Un rassemblement régional le 7 avril 1935 à Saint-Brieuc, animé par le député de Gironde Philippe Henriot, déplace 2500 personnes mais on en attendait le double. Ce meeting d'un futur responsable des jeunesses de la fédération républicaine (JFR) permet néanmoins de mettre en évidence les convergences entre la droite catholique et les ligues. Nombre de catholiques d'Action française qui se sont soumis aux consignes de Rome vont rejoindre le PSF après 1936. En 1939, l'AF a disparu des Côtes-du-Nord - il reste alors six personnalités fichées comme telles.

27. SAINCLIVIER, Jacqueline, op. cit., p. 78-79.

28. Ibidem, p. 89-90 et BOUGEARD, Christian, thèse citée, p. 364-373 sur les ligues. Une liste nominative de 1937 ou 1938 donne les noms de 56 membres de l'AF dont 64,3\% de nobles. Deux deviennent des responsables du PSF.

29. Il faudrait une étude de la Loire-Inférieure.

30. THOMAs, Jean-Paul, « Les effectifs du parti social français ", Vingtième siècle, n ${ }^{\circ} 62$, avril-juin 1999, p. 61-83. Carte III d'après Jean Philippet. 
Les Croix-de-Feu apparaissent après le 6 février 1934 et en 1935 lors de réunions urbaines. Des sections tentent de se constituer : à Saint-Brieuc, elle tombe en léthargie au printemps 1935, à Dinan (de 20 à 40 affiliés), à Rennes (une soixantaine avec les Volontaires nationaux). Dans les régions de Vannes et de Dinan, les Croix-de-Feu recrutent dans les milieux de la droite " réactionnaire et cléricale " (sous-préfet de Dinan) et souvent chez d'anciens officiers nobles. Pour les autorités, ce mouvement et les ligues sont un moyen de regagner le terrain politique perdu face aux radicaux en 1932. Le Finistère présente une situation particulière car les Croix-de-Feu s'y développent dès 1933. La section de Quimper passe de 12 adhérents le 14 juillet 1933, à 120 à la fin de $1934^{31}$. En juillet 1934, celle de Landerneau compte déjà environ 200 membres. Il faut dire que cette cité est celle de l'hôtelier Eugène Leclerc (commandant de réserve), délégué régional pour la Bretagne ${ }^{32}$. De quatre sections (avec Brest et Morlaix) et 420 militants à la fin 1934, on passe à huit (et de nombreuses sous-sections dans la région brestoise et le Léon) et un minimum de 3000 adhérents en juin 1936, plus 500 membres des sections d'action féminine et sociale. C'est un véritable mouvement de masse qui se met en place dans le Finistère nord face à la montée en puissance du Front populaire. Dans les Côtes-du-Nord, à partir de l'automne 1935, les Croix-deFeu se réorganisent (Saint-Brieuc), intensifient leur propagande (500 personnes à Dinan, 350 à Saint-Brieuc) et posent des jalons dans l'Ouest sous l'impulsion de F. Touche, un propriétaire agriculteur élu maire de Trélévern en 1935, venu de l'AF et futur responsable départemental du PSF.

Sauf dans le Finistère et la Loire-Inférieure, les ligues de la droite extrême ont peu d'impact en Bretagne avant 1936. En revanche, la crise permet aux droites d'exploiter les mécontentements avec deux types de mouvements : la Fédération ou ligue des contribuables et le Front paysan instrumentalisés par Henri Dorgères. Dorgères est le délégué régional et le président de cette ligue installée à Rennes, dans les locaux de son propre journal Le Progrès Agricole de l'Ouest ${ }^{33}$. C'est en février et mars 1933 que la ligue des contribuables organise de grands rassemblements dans les villes de la France de l'Ouest où Henri Dorgères prend la parole, à Dinan (19 février et 4 mars, 2500 personnes), Gourin (26 février, 450 personnes mais 30 adhésions), Rennes (24 mars, 4000), Nantes (10000). Hors d'Ille-et-Vilaine, où 45 " syndicats " existent, le tribun démagogue Dorgères dénonce « la gabegie de l'État " et les fonctionnaires cumulards, se faisant d'abord connaître sous la casquette de la ligue des contribuables ${ }^{34}$. Des élus de la droite répu-

31. Le Roux, Bruno, Le mouvement Croix-de-Feu et le PSF dans le Finistère, M. H., Brest, UBO, 1996, p. 7-15.

32. Instituteur dans le Doubs, agrégé d'histoire, quatre fois blessé, Édouard Leclerc se marie et s'installe à Landerneau en 1919. C'est le père de quinze enfants dont Édouard Leclerc, le créateur de la chaîne de magasins.

33. Pedrono, Maryvonne, Le Morbihan au temps des fourches. Le dorgérisme dans le Morbihan dans les années 1930, M. H., Rennes 2, 1996. Selon un rapport de police, les trois autres membres du bureau appartiennent à l'AF et à l'extrême droite, p. 37-42.

34. D'autres réunions ont lieu dans le Finistère et le Morbihan sans Dorgères (2000 personnes à Pontivy le 6 février 1933). 
blicaine y participent comme le député de Kéroüartz qui est présent lors de la mise sur pied d'un bureau départemental à Saint-Brieuc. Mais ce mouvement qui s'appuie sur le mécontentement des classes moyennes et de la paysannerie retombe en 1935 lorsque la situation économique s'améliore, et aussi parce que des forces politiques structurées prennent le relais.

Le dorgérisme et le Front paysan tendent aussi à se confondre même s'il y a quelques conflits de pouvoir entre la droite traditionnelle et le courant démagogique et fascisant, pour ne pas dire fasciste, incarné par Dorgères lui-même à partir de la formation des Chemises vertes qui tiennent leur premier congrès à Bannalec (Finistère) en 1935. L'Ille-et-Vilaine, où Henri Dorgères lance ses premiers comités de Défense paysannne en 1929 (une quarantaine de réunions) contre la loi sur les assurances sociales et où il réunit 16000 paysans à Rennes le $1^{\text {er }}$ février 1930, reste la base du mouvement jusqu'à la guerre (313 réunions dorgéristes au total) ${ }^{35}$. Ce n'est qu'à partir de 1933 que l'agitation dorgériste gagne les autres départements bretons et ceux de l'Ouest intérieur. Elle ne touche que faiblement la bordure orientale des Côtes-du-Nord, mais se développe dans le Morbihan et dans le Finistère où elle trouve des relais importants dans la paysannerie, chez certains notables ou parmi les cadres des grands syndicats-boutiques conservateurs (Union des syndicats agricoles de Bretagne méridionale et Office central de Landerneau) qui s'efforcent ainsi de canaliser le mécontentement paysan. Ils sont 8000 à 12000 à Quimper le 29 janvier 1933. Mais dans les régions bleues de la Montagne finistérienne, de la Haute-Cornouaille et du Trégor, des militants paysans socialistes de la CNP conduits par Tanguy Prigent et de la CGPT (socialistes et communistes) s'opposent aux ventes-saisies de 1932 à 1936 et disputent victorieusement le terrain à Dorgères.

Dans le Morbihan, des notables de droite s'investissent dans le mouvement paysan. L'ancien député de Pontivy-1 Joseph Cadic (battu en 1932 par le radical-socialiste Lotz) a adhéré au parti agraire et paysan français (PAPF) de Fleurant-Agricola et il organise les premières réunions agraires (6 en 1933, 2 en 1934) en appelant " au nettoyage politique " de l'Assemblée nationale ${ }^{36}$. Sa fédération participe au congrès national du parti qui s'achève par une scission le 4 février 1936 car les dorgéristes ont cherché a en prendre le contrôle. Auparavant, J. Cadic a participé avec Dorgères et de nombreux aristocrates conservateurs aux deux grands meetings pour vanter les mérites du Front paysan lancé en juillet 1934. Le 25 mars et le 19 mai 1935, à Josselin (3000 à 4000 personnes) et à Questembert (3000), toutes les droites et les extrêmes droites anciennes et modernes sont réunies sur la même tribune. Mais ensuite, J. Cadic qui a sa propre ligue de défense paysanne, entre en conflit avec les dorgéristes qui, à son goût, attaquent trop durement le PDP et les républicains modérés à l'approche des élections législatives de 1936.

35. Fricot, Régis et Genaitay, Pierre, Dorgères et le mouvement paysan, M. H., Rennes, 1972. 36. PEDRONo, Maryvonne, op. cit., p. 53-74. 
En fait, de 1934 à 1936, le dorgérisme prend un certain essor dans les campagnes grâce au ralliement d'authentiques militants paysans venus du syndicat des " cultivateurs-cultivants " (FSPO) qui disparaît en 1933 dans la tourmente. Ainsi Jean Bohuon devient l'un des principaux lieutenants de Dorgères (président du Comité de défense d'Ille-et-Vilaine en 1935), de même que Jean Coirre ou Jean Nobilet. Dans le Finistère, ce sont les frères Divanac'h liés à Landerneau; dans le Morbihan Pierre Bléher, riche propriétaire exploitant et Le Pêcheur, président départemental.

La montée du mécontentement politique et social en 1934 n'est pas sans retombées électorales lors des élections cantonales d'octobre 1934. Ainsi, dans les Côtes-du-Nord, où la stabilité l'emporte (19 notables sortants sur 24 sont réélus), les radicaux-socialistes perdent deux sièges au profit de modérés (radicaux indépendants) ${ }^{37}$. À Dinan-est, Michel Geistdoerfer est battu par l'URD Thoreux à l'issue d'un affrontement très rude. Le candidat de droite a fait campagne sur des thèmes nationalistes en approuvant à la fois les émeutiers du 6 février 1934 et le gouvernement d'union nationale de Gaston Doumergue : «Le 6 février le peuple s'est réveillé. Il a chassé les profiteurs cartellistes et imposé Doumergue ", lequel « issu des manifestations qualifiées faussement d'émeutes est notre dictateur légal et la France s'en trouve bien ". Les gouvernements radicaux-socialistes sont accusés de " prendre [leurs] mots d'ordre aux loges maçonniques et à l'étranger $^{38}$ ". Dans la région dinannaise devenue le lieu des rassemblements des droites extrêmes en 1933-1934 (dorgérisme, Ligue de contribuables, Croixde-Feu, Comité anticollectiviste), une droite dure s'organise sur les débris de l'Alliance démocratique avant même le Front populaire.

C'est dans ce climat d'affrontement bloc contre bloc que se déroulent les élections législatives de 1936, ce qui rejette le PDP à droite. Les candidats de droite et du centre agitent le chiffon rouge de l'anticommunisme. Montfort, le futur député " paysan " de Quimperlé, avance son slogan : " Famille, métier, Patrie " quand le $\mathrm{D}^{\mathrm{r}}$ Joly, élu à Rennes-2 lui répond par " honneur, probité, travail, famille, patrie ". Le PDP Le Guen, battu à SaintBrieuc-1, fustige les candidats " du désordre, du gâchis, de la guerre civile, préface à la guerre étrangère ». La droite présente un candidat unique dans 29 circonscriptions sur 44. Mais il est intéressant de souligner que des candidats " agraires " cherchent à abattre les notables dans certains fiefs de droite. À Vitré, le dorgériste Jean Bohuon tente en vain de battre le député sortant conservateur, le vicomte de Lyrot ${ }^{39}$. À Brest-2, le PDP Paul Simon, réélu au second tour, a dû affronter pour la première fois Uchard qu'il présente dans ses tracts comme le candidat de "tous les ennemis du Régime", l'homme de Dorgères " partisan de l'Action française ", " de la violence et de l'illégalité ". " Contre le fascisme ", le démocrate populaire appelle à voter " pour la République ".

37. La droite gagne aussi trois sièges au conseil d'arrondissement.

38. BougEARD, Christian, thèse citée, p. 347-349.

39. SAINCLIVIER, Jacqueline, op. cit., p. 46. 
Les scrutins sont souvent serrés avec parfois des triangulaires défavorables au Front populaire ${ }^{40}$. Avec 20 sièges en Bretagne (9 URD, 11 républicains indépendants et d'action sociale et agraire) les droites font une percée importante alors que le PDP (6 élus) ne gagne qu'un siège à SaintBrieuc-2 où Alfred Duault l'emporte sur le radical sortant Cornu. Pierre Trémintin dans le Léon, Ernest Pezet (68,6 \%) et le chanoine Desgranges $(59,8 \%)$ dans le Vannetais sont réélus dès le $1^{\text {er }}$ tour sans adversaires à droite. En dehors de ses bastions, le centrisme démocrate-chrétien ne parvient pas à s'imposer. L'ARD et les radicaux indépendants hostiles au Front populaire sont laminés (quatre députés).

Une droite catholique paysanne, constituée de véritables exploitants agricoles proches ou membres de la Fédération républicaine, émerge, ainsi qu'une nouvelle génération de jeunes élus beaucoup plus militante ${ }^{41}$. Des notables traditionnels de droite, déjà âgés, sont bousculés par des nouveaux venus comme Pierre Gillet (39 ans) à Ploërmel ${ }^{42}$. Le renouvellement des générations se fait aussi à Quimper-1 avec Hervé Nader (36 ans) ainsi qu'à Pontivy-2 où Paul Ihuel (32 ans) bat le socialiste Le Coutaller qui a distancé au $1^{\text {er }}$ tour le député radical-socialiste sortant. Paul Ihuel entame une longue carrière politique comme le notaire Jean Crouan (30 ans), élu maire en 1935, qui bat l'ancien ministre de la gauche radicale Charles Daniélou ${ }^{43}$. S'étant présenté comme républicain modéré, proche des Croix-de-Feu, Jean Crouan s'inscrit à l'URD et la Fédération républicaine sur les conseils de son oncle Jules Fortin, ancien sénateur du Finistère ${ }^{44}$. Mais le député finistérien souhaite la constitution d'un groupe unique de droite y compris avec les huit députés qui rejoignent le PSF. Crouan et Nader vont adhérer au Comité parlementaire de défense des libertés républicaines et de sympathie pour le PSF (59 membres), ce qui traduit bien leur proximité avec cette droite musclée qui émerge ${ }^{45}$. Au congrès de Nice, en 1936, Jean Crouan participe aussi à la fondation puis au comité directeur, avec François Valentin, des Jeunesses de la fédération républicaine (JFR) présidées par Philippe Henriot ${ }^{46}$. La vic-

40. À Quimper-1, le RS sortant Pouchus se maintient et permet l'élection d'Hervé Nader contre le communiste P. Guéguin, maire de Concarneau.

41. Cadic est réélu à Pontivy. C'est le cas de L. Montfort à Quimperlé, de P. Lohéac à Châteaulin-2 contre le SFIO Masson, de Y. Hervé à Guingamp-1 contre le maire de Guingamp Lorgeré (RS).

42. Au $1^{\text {er }}$ tour P. Gillet (URD) distance le $D^{r}$ Guillois (URD) et il bat au second le conservateur Jean du Plessix de Grénédan. Il n'a pas respecté l'accord proposé par l'Action catholique qui prévoyait le maintien du seul candidat de droite arrivé en tête.

43. CrouAN, Erwan, Jean Crouan 1906-1985. Un notable finistérien dans la tourmente du xxe siècle, M. H., Brest, UBO, 2001, p. 23-35.

44. JEANNENEY, Jean-Noël, "La fédération républicaine ", La France et les Français en 19381939, REMOND, René et BouRdin, Janine (dir.), PFNSP, 1978, p. 345-346.

45. Nobecourt, Jacques, Le colonel de La Rocque 1885-1946 ou les pièges du nationalisme chrétien, Fayard, 1996, p. 643-644.

46. BIGORGNE, Laurent, "Le parcours politique d'une génération de "modérés" : les jeunes de la Fédération républicaine ", Les modérés dans la vie politique française, op. cit., p. 385-396. 
toire nationale du Front populaire va stimuler l'opposition des droites parlementaires mais aussi réactiver les opposants issus des ligues.

\section{Durcissement et reclassements des droites en Bretagne contre le Front populaire et à l'approche de la guerre}

Deux approches permettent de mesurer le poids des droites en Bretagne à la veille de la guerre : d'une part l'impact des mouvements non-parlementaires et souvent antiparlementaires, de l'autre l'enracinement électoral local qui permet de mesurer l'évolution des rapports de force. Dès l'été 1936, l'agitation dorgériste reprend avec une volonté de politisation évidente. Le 28 juin 1936, Dorgères et Bohuon tiennent des réunions dans cinq localités du Morbihan et il y en aura 36 jusqu'à la fin de l'année, mais elles mobilisent peu (20 à 150 personnes), sauf quand le chef se déplace lui-même (3 fois $)^{47}$. Le 22 novembre, à Muzillac, le PSF apparaît comme l'organisateur. Il s'agit souvent de créer des comités de défense paysanne. Le mouvement en revendique 200 et " 15000 paysans mobilisables " en mars $1938^{48}$. Le principal orateur est Bohuon (46 réunions de 1935 à 1939). En 1937, 31 réunions sont recensées par les autorités dont un rassemblement de 12000 personnes à Languidic le 11 juillet, puis 40 en 1938, presque toutes avant l'été. Les auditoires sont beaucoup plus limités. Le congrès interdépartemental du Faouët le 3 juillet 1938, avec défilé des Chemises vertes au monument aux morts malgré l'interdiction, n'a déplacé que 3000 à 4000 personnes alors qu'on en attendait 10000. La fin du Front populaire fait retomber la pres$\operatorname{sion}^{49}$. Dans les Côtes-du-Nord, les dorgéristes tiennent huit réunions au premier semestre 1937 mais, selon le préfet, ils ne rencontrent plus " aucun écho parmi les ruraux ". Un certain déclin se fait aussi sentir en Ille-et-Vilaine en 1937 et en 1938 malgré le premier congrès régional de l'unité paysanne qui rassemble 20000 paysans à Rennes le 3 avril 1938. L'amélioration économique et sociale de la politique du Front populaire (Office du blé) désamorce largement en Bretagne la grogne paysanne.

La création par le colonel de La Rocque du Parti social français à partir des Croix-de-Feu le 12 juillet 1936, va engager une organisation partisane et militante d'un parti de droite bien structuré en Bretagne. La dissolution des ligues par le gouvernement Blum le 18 juin n'a provoqué que peu de réactions ${ }^{50}$. Le PSF développe des sections dans les villes d'Ille-et-Vilaine (sauf à Vitré) où il aurait 1500 à 2000 adhérents en $1938^{51}$. L'influence du PSF qui recrute chez les conservateurs paraît assez limitée chez les notables et les élus de l'arrondissement de Vannes ${ }^{52}$. Il s'organise dans les Côtes-

47. PEDRONO, Maryvonne, op. cit., p. 105-117.

48. Le Nouvelliste du Morbihan, 30 mars 1938.

49. Il y aura encore 5 réunions dans le Morbihan en janvier 1939.

50. Le 21 juin, on signale des petites réunions à Landerneau et Saint-Julien (Côtes-duNord) et 91 personnes brandissant des drapeaux tricolores à Dinan.

51. SAINCLIVIER, Jacqueline, op. cit., p. 93.

52. RIVIERE, Christophe, Notables municipaux et élus départementaux en Morbihan 19321959, M. H., Brest, UBO, 1998, p. 13-14 mais une étude précise serait nécessaire. 
du-Nord en septembre et octobre 1936 sous la houlette de Graziani, le responsable de la section de Guingamp. Par des réunions privées dans les campagnes et les petites villes, avec l'appui d'une partie du clergé et de certains notables URD, le PSF recrute dans le patronat et les classes moyennes indépendantes. À Pontrieux, il a l'appui du Front paysan dorgériste et des Chemises vertes ${ }^{53}$. Avec Eugène Leclerc, le délégué régional, le PSF organise un banquet de 250 convives à Saint-Brieuc le 29 novembre 1936. Mais la mobilisation reste finalement assez limitée dans ce département orienté à gauche (19 réunions en 1937, 14 en 1938, 5 en 1939; de 100 à 250 auditeurs). Elles sont concentrées dans la région de Dinan et dans le Trégor qui a élu le député socialiste Philippe Le Maux. En juin 1937, Charles Vallin tient un grand meeting à Saint-Brieuc (1500 personnes) suivi par Jean Ybarnégaray à Lamballe en juillet (2500). Ensuite, l'activité militante du PSF paraît s'essouffler au moment où F. Touche, président départemental, organise une fédération au début de $1938^{54}$. Dans ce département, les forces de droite, hormis le PDP qui tient son congrès à Perros-Guirec le 11 avril 1937 (400 personnes), ont bien du mal à organiser des structures permanentes. Avec la mort de Le Trocquer, l'ARD semble avoir disparu et, malgré deux députés proches, la Fédération républicaine est à réorganiser en 1939 comme en Ille-et-Vilaine ${ }^{55}$.

La situation du PSF finistérien est quelque peu différente. À sa naissance, il bénéficie de l'appui du journal catholique conservateur, Le Courrier du Finistère $^{56}$. Son essor est rapide et considérable : en 1937, il compte 20 sections avec un bureau et 45 délégations locales situées aux deux tiers dans le Finistère nord ${ }^{57}$. Au début de 1937, le PSF finistérien aurait 12000 adhérents, près de quatre fois plus que le PCF et la SFIO réunis. Mais ses effectifs stagnent après la crise que traverse la direction nationale (affaire Pozzo di Borgo) et des dissidents fondent même en mai 1938 une section du Parti populaire français (PPF) de Doriot à Landerneau $(25 \text { membres })^{58}$. Eugène Leclerc a été nommé par le colonel Jacques de La Rocque délégué régional du parti, chargé de suivre et d'organiser la propagande des trois autres fédérations bretonnes (sans la Loire-Inférieure). La capacité de mobilisation du PSF est forte : 10000 lors de quatre meetings de La Rocque le 20 décembre 1936; 3000 en mai 1937 à Brest pour Vallin puis 1500 à SaintPol-de-Léon dont le maire et conseiller général est Alain Budes de

53. BOUGEARD, Christian, thèse citée, p. 520-525.

54. Rien dans les sources départementales n'indique que le PSF aurait eu entre 5000 et 10000 adhérents en 1937 dans les Côtes-du-Nord (et en Ille-et-Vilaine) comme l'estime Jean-Paul THOMAs, article cité, carte I.

55. JEANNENEY, Jean-Noël, op. cit., carte p. 342. Les deux députés URD Morane et Hervé sont inscrits au groupe des républicains indépendants et d'action sociale.

56. LE RouX, Bruno, op. cit., p. 18-67.

57. Le PSF a une permanence à Brest et la fédération est présidée par Jean Richard, ancien responsable des Croix-de-Feu de Morlaix.

58. Un groupe du PPF apparaît aussi à Saint-Brieuc en 1937 (12 adhérents), animé par des conservateurs. Mais il reste à l'état groupusculaire malgré des meetings de Doriot qui vient en vacances au Val-André. 
Guébriant, le fils du dirigeant de Landerneau; 4000 dans la même ville pour Ybarnégaray en août 1937. En février et mars, une quinzaine de réunions du PSF sont organisées dans le Finistère sud dont une à Quimper avec le député de droite Nader qui est un sympathisant notoire ${ }^{59}$. Elles sont parfois houleuses, notamment dans les ports, car la gauche et les communistes y apportent la contradiction. Le 6 août 1939, La Rocque déplace encore au moins 32000 personnes à une fête à Clohars-Fouesnant (Finistère sud). L'organe régional, La Volonté Bretonne, dont le rédacteur en chef est le Brestois Pierre Branellec, paraît du 5 octobre 1936 à mai 1940 et tire à 25000 exemplaires. Les cadres du parti se recrutent dans la petite bourgeoisie; quelques uns ont des responsabilités à l'Office central de Landerneau et l'UNC fournit des troupes. Comme pour le dorgérisme, la droite conservatrice préfère accompagner le PSF plutôt que de s'y opposer ${ }^{60}$. Le PSF a pour ambition d'encadrer la société avec ses œuvres sociales (un service d'entraide, des sections féminines et d'action sociale), ses activités festives et artistiques. Contre la CGT, il encourage la création de syndicats professionnels français de la CSPF (dans le Finistère et les Côtes-du-Nord) ainsi que des aéro-clubs Jean Mermoz à Quimper et à Morlaix. Le PSF apparaît bien comme une force majeure dans la recomposition des droites finistériennes de 1936 à $1940^{61}$. Plus que des rivalités, ce sont des passerelles ou des proximités qui semblent exister dans ce département.

La droite conservatrice et catholique n'est pas en reste. Jean Crouan, " PSF de cœur et Fédération républicaine de raison ${ }^{62}$ ", organise ainsi le 14 février 1937 à Pleyben une grande manifestation de la droite nationale finistérienne (7 députés ou anciens députés, 7 conseillers généraux, les responsables de l'UNC et des républicains nationaux). 15000 personnes (6000 selon la police) viennent écouter les harangues xénophobes, antijacobines voire antisémites de Philippe Henriot, Xavier Vallat et Emmanuel Temple, des " jeunes loups " de la Fédération républicaine ${ }^{63}$. Le banquet réunit 2000 convives tandis que le Front populaire a mobilisé 4000 contremanifestants. La radicalisation politique touche aussi la droite catholique, ce qui place le PDP absent de plus en plus en porte-à-faux.

Ces évolutions vers la droite de l'échiquier politique ont-elles un impact sur les élus locaux bretons dans la deuxième moitié des années 1930? Ayant glissé d'un positionnement centriste, du fait de son attachement à la laïcité au début des années 1930, vers la droite de 1934 à 1938, l'Alliance républicaine démocratique tend à être laminée sur sa gauche par les radicaux indépendants et les radicaux-socialistes et sur sa droite par l'affirmation de la

59. Des réunions se tiennent en breton et se terminent avec l'hymne breton, le Bro Goz Ma Zadou.

60. Un syndicat du PSF compte 109 adhérents parmi les employés de l'Office de Landerneau.

61. Nous n'avons pas d'éléments sur le Morbihan ni sur la Loire-Inférieure, zone de force du PSF.

62. L'expression est de son petit-fils Erwan CROUAN, op. cit., p. 41.

63. Ibidem, p. 35-36. 
Fédération républicaine-URD et la montée en puissance du PSF qui prend le relais à droite des conservateurs. Cette évolution est très nette dans les Côtes-du-Nord sans que le sénateur Le Trocquer parvienne à l'enrayer. Les républicains de gauche (ARD) passent de cinq conseillers généraux en 1934 à trois en 1937 alors que ceux de l'URD progressent de deux à cinq et que les conservateurs sont en voie de disparition (de six à trois) ${ }^{64}$. L'évolution a été comparable au niveau municipal de 1925 à 1935, même si les forces modérées et de droite contrôlent encore 51,2 \% des municipalités en 1935. Les conservateurs ont reculé de 90 à 39 municipalités (10\%); les républicains de gauche se tassent, de 87 à 66 (16,9\%) alors que l'URD progresse légèrement passant de 83 à 88 communes $(22,5 \%)^{65}$. Malgré leur recul en 1935, les notables de droite (le PDP dirige 7 communes, 1,8 \%) résistent bien, localement, dans les campagnes de ce département où la gauche dirige les villes et les chefs-lieux de canton. Le PSF ne parvient pas à s'implanter (4 maires, $1 \%$ ).

Qu'en est-il du Finistère? Dans le Léon, les droites dominent sans partage la vie municipale tout au long des années 1930, en dehors de l'agglomération brestoise et de Landerneau ${ }^{66}$. Dans l'arrondissement de Brest, en 1929 et en 1935, elles contrôlent 69 (81 \%) puis 65 des 85 communes dont 26 pour les conservateurs et 38 pour l'URD en $1935^{67}$. Nous manquons de données globales pour l'ensemble du département ${ }^{68}$. Dans cette région qui envoie portant deux députés démocrates-chrétiens au Palais-Bourbon, le PDP ne détient qu'une seule mairie, quatre dans l'ensemble du Finistère, et quatre conseillers généraux sur 43 dont trois dans le Léon en 1937. Dans le sud, l'arrondissement de Quimper plus à gauche compte en 1935, 10 \% de maires avec l'étiquette ARD et 36,9 \% URD. Malgré son dynamisme, le PSF n'a attiré que sept maires (2,3\%, 6 nobles) du Finistère, élus comme conservateurs en 1935, et deux conseillers généraux léonards ${ }^{69}$.

Autre pays de tradition blanche et de chouannerie, l'arrondissement de Vannes présente une domination municipale des droites qui tiennent $71,4 \%$ des mairies. En 1935, sur 123 communes les conservateurs en contrôlent 26 (21,1 \%), la Fédération républicaine 52 (42,2 \%) et le PDP 10

64. BOUGEARD, Christian, thèse citée, p. 538. Un militant du PSF est élu au conseil d'arrondissement à Lannion.

65. Ibidem, p. 351-353.

66. En 1929, une liste conduite par Victor Le Gorgeu (radical), élu sénateur en 1930, allie radicaux et modérés pour enlever la ville de Brest à la SFIO.

67. QuinTIN, Virginie, Élus municipaux et cantonaux de l'arrondissement de Brest (19341951), M. H., Brest, UBO, p. 17-20. Dans cette " terre des prêtres " cléricale, les laïcs centristes (8 mairies pour les républicains de gauche et 4 pour les radicaux indépendants) sont perçus de gauche. La SFIO en a 2 (Lambézellec et Landerneau) et les radicaux-socialistes 3.

68. Une statistique de 1929 sur les étiquettes des conseillers municipaux donne 47,2\% pour les droites (24,5\% pour les conservateurs, 22,7 \% pour l'URD). Les centristes républicains de gauche en ont $16,2 \%$.

69. Il s'agit de Boucher de Ploudiry et de De Taine de Saint-Renan qui sera nommé président de la commission administrative par Vichy. 
$(8,1 \%)^{70}$. À droite, une relève est en train de s'opérer entre les conservateurs en perte de vitesse (30,8\% des maires à la veille des élections) et une droite nettement républicaine et parlementaire qui passe de $38(30,8 \%)$ à 52 mairies. Si le PDP contrôle quelques municipalités, son implantation locale est sans commune mesure avec sa représentation parlementaire puisque, depuis 1928, deux PDP, Ernest Pézet et l'abbé Desgranges (apparenté), sont élus dans les deux circonscriptions du Vannetais. En 1937, les deux cantons de Vannes comptent d'ailleurs les deux seuls conseillers généraux PDP du Morbihan. En Ille-et-Vilaine, la droite s'est renforcée aux élections municipales de 1935 face à un centre gauche modéré ${ }^{71}$.

Les élections cantonales d'octobre 1937 puis les sénatoriales d'octobre 1938 sont des repères importants des rapports de force politiques car elles sont nettement politisées entre partisans et adversaires du Front populaire. Dans les Côtes-du-Nord, la majorité radicale du conseil général se consolide alors qu'elle s'effrite dans le Finistère. Dans ce département, la majorité de la gauche laïque du sénateur Lancien (radical) tend d'ailleurs à se réduire : 23 élus contre 20 à la droite (7 conservateurs, 9 URD, 4 PDP). Elle ne tient qu'avec l'appui de quatre républicains de gauche qui dans ce département n'ont pas encore évolué vers la droite ${ }^{72}$. Le conseil général d'Ille-et-Vilaine fait aussi l'objet depuis 1919 de combats politiques acharnés pour plus de la moitié des cantons ${ }^{73}$. En 1928, les républicains laïques le reconquièrent avec une voix de majorité mais ils le perdent en 1937 avec le même écart. Le sénateur-maire radical-socialiste de Saint-Malo, Alphonse Gasnier-Duparc, président du conseil général depuis 1935 et ministre de la Marine de Léon Blum, doit laisser son fauteuil à Marcel Rupied, maire de droite de Vitré. Signe de durcissement politique, trois candidats du PSF se présentent et deux sont élus, à Redon et à Maure-de-Bretagne, les frères Lagrée. L'évolution est identique dans le Morbihan où le conseil général, auparavant présidé par un radical, bascule à droite en faveur du $\mathrm{D}^{\mathrm{r}}$ Guillois (URD), même si le rapport des forces a peu bougé ${ }^{74}$.

À la veille de la guerre, la poussée à droite est sensible en Bretagne lors des élections cantonales de 1937 (sauf dans les Côtes-du-Nord) mais aussi lors des sénatoriales d'octobre 1938. Dans les Côtes-du-Nord, toutes les droites se retrouvent sur deux modérés, le Dr Bouguen (républicain de gau-

70. RIVIERE, Christophe, Notables municipaux, op. cit., p. 11-12. L'URD était majoritaire dans 1/3 des communes du Morbihan en 1932. En 1935, les laïcs ne dirigent que 28,4\% des municipalités de l'arrondissement de Vannes (17 républicains de gauche et 18 radicaux dont Vannes).

71. SAINCLIVIER, Jacqueline, op. cit., p. 70-73 et carte $\mathrm{n}^{\circ} 10$.

72. La majorité se compose de 1 PCF, 5 SFIO, 1 rép. soc., 9 rad.-soc., 3 rad. ind., 4 rép. de g. 73. SAINCLIVIER, Jacqueline, op. cit., p. 55-66.

74. RiviERE, Christophe, op. cit., p. 54. Les conservateurs (quatre sièges) et le PDP (deux) restent stables alors que l'URD en gagne un (onze) ainsi que les républicains de gauche (six). Ce sont eux qui font basculer la majorité alors que les radicaux sont passés de 15 à 13 et que la SFIO a deux élus à Lorient. En 1932, la liste radicale avait remporté les quatre sièges de sénateurs. 
che) et le maire de Lannion Edgar de Kergariou (républicain indépendant), pour éliminer les radicaux-socialistes partisans du Front populaire dont Meunier, président du conseil général et sénateur sortant ${ }^{75}$. Les rivalités de personnes chez les vieux notables de gauche se réclamant du radicalisme et l'arbitrage de la droite ont fait pencher la balance en faveur de trois modérés contre deux radicaux ${ }^{76}$. Mais à quelques exceptions près, le PSF ne parvient à prendre pied. Le Finistère évolue dans le même sens puisque la droite obtient trois élus pour seulement deux radicaux sortants réélus (les docteurs Lancien et Le Gorgeu). Trois des cinq candidats "nationaux " désignés au congrès du Faou le 23 septembre 1938 l'emportent : Queinnec, le nouveau maire de Morlaix Le Jeune, et celui de Ploaré, du Frétay. Gabriel Queinnec, notaire à Pont-L'Abbé, ancien député URD de 1928 à 1932, avait déjà gagné l'élection partielle du $1^{\mathrm{er}}$ mai 1937 avec l'appui de Jean Crouan en succédant à son vieil adversaire radical-socialiste Georges Le Bail décédé. Le PDP qui soutenait la liste de droite en 1929 propose cette fois une liste de concentration républicaine en accord avec sa position nationale d'appui au gouvernement d'Édouard Daladier (les deux radicaux élus et deux hommes de droite Inizan et Quéinnec) ${ }^{77}$. Cette position provoque une crise dans un parti déjà en déclin : l'ancien conseiller général Pierre Mocaër voulait une liste de droite avec deux PDP et, comme il a été désavoué, il se présente seul. Le positionnement centriste est bien difficile à tenir dans une période où la droite la plus dure a le vent en poupe dans l'ensemble de la Bretagne depuis 1936.

Une ultime élection législative partielle dans les Côtes-du-Nord, en avrilmai 1939, à Saint-Brieuc-1, permet de mesurer les évolutions politiques au sein des droites. Il s'agit de remplacer le radical-socialiste Pierre Michel entré au Sénat. Au $1^{\text {er }}$ tour, le PSF Louis Huchet du Guermeur (24 \%) devance Émile Le Guen (18,9 \%) qui représente le PDP depuis $1932^{78}$. Au second tour, contre le RS François Auffray, l'homme du PSF a l'appui de toutes les droites y compris du député PDP de droite Alfred Duault, mais pas de Le Guen dont la moitié de l'électorat va voter radical. Huchet du Guermeur n'en est pas moins très nettement battu (34,8 \%) dans une circonscription enracinée à gauche. Si le PSF bénéficie en Bretagne comme ailleurs d'un développement militant important de 1936 à 1939, son implantation électorale reste très limitée.

Dans les années 1930, la droite évolue en Bretagne à la faveur de l'agitation sociale de la Grande Dépression et de la victoire électorale du Front populaire. D'abord, les centristes laïques de l'ARD sortent affaiblis d'une polarisation gauche-droite très marquée, et une partie de ses cadres évo-

75. Précisons que Meunier avait commencé sa carrière comme député de droite (Alliance libérale populaire) en 1913 et avait rejoint les républicains puis les radicaux après 1919. Le radical indépendant Betfert qui avait succédé à Le Trocquer est réélu.

76. BOugEARD, Christian, thèse citée, p. 551-554.

77. Floch, Jean-Charles, op. cit., p. 206-213.

78. Bougeard, Christian, thèse citée, p. 554-557. 
lue clairement vers la droite dans l'opposition à un radical-socialisme qui renouvelle ses députés et se positionne à gauche (dans les Côtes-du-Nord et à Dinan). Le PDP se consolide dans ses bastions électoraux en supplantant les notables conservateurs et souvent aristocrates (Léon, Vannetais) mais sans parvenir à s'implanter localement. Il doit donc compter sur des élites locales et un électorat de droite catholique, et il ne parvient pas à s'affirmer réellement comme un nouveau centre républicain. Même s'ils résistent localement (Loire-Inférieure, Vannetais, Vitréen), les grands notables conservateurs héritiers de la tradition monarchiste et de l'Action française doivent céder du terrain au profit d'une nouvelle droite plus paysanne, issue ou proche de la Fédération républicaine. Le mouvement est d'autant plus net dans les élections de 1935 à 1937 que l'URD est capable de faire émerger ou d'attirer à elle de jeunes députés à côté de ses notables ruraux. L'agitation agraire et dorgériste est importante dans la région en 1933-1935 où Dorgères et ses lieutenants peuvent jouer les trublions antiparlementaires et fascisants. Mais, malgré son activisme de 1936 à 1938 contre le Front populaire (dans le Morbihan et le Finistère), le dorgérisme mobilise beaucoup moins de monde dans les campagnes bretonnes et il doit souvent faire face à l'opposition des militants de gauche. L'enracinement de cette extrême droite est beaucoup moins profond que sa courbe d'activités ne le laisserait penser. L'impact du parti national breton (PNB), pris en main à partir de 1937 par Olier Mordrel et Fransez Debauvais, deux leaders attirés par le nazisme, est trop faible pour peser dans la vie politique, tout comme le PPF. Mais ces deux courants vont se manifester dans le collaborationnisme sous l'Occupation. Sauf dans le Finistère et en Loire-Inférieure où une activité importante et une attraction militante des classes moyennes se déploient de 1936 à 1939, le PSF ne parvient pas véritablement à s'imposer sur le terrain électoral. En effet, si une partie des responsables de la droite conservatrice accompagne le mouvement en 1936 et 1937, très peu d'élus s'y rallient finalement, et le PSF ne parvient pas à s'imposer dans les urnes en 1937 et 1938. Le 10 juillet 1940, tous les parlementaires bretons, de gauche comme de droite présents à Vichy, votent les pleins pouvoirs au maréchal Pétain sauf sept Finistériens ${ }^{79}$. Plusieurs notables de droite deviennent des pétainistes actifs et acceptent de siéger au Conseil national à Vichy (Crouan, Nader, Rupied, Duault). Hervé Budes de Guébriant et Dorgères sont des chefs de la Corporation paysanne. Mais certains entrent rapidement en résistance et seront déportés en Allemagne (Crouan, Nader) ${ }^{80}$. Beaucoup de maires s'efforcent de protéger leurs administrés face aux exigences allemandes. Une nouvelle période s'ouvre pour la droite bretonne.

79. Deux SFIO, deux radicaux-socialistes, un radical et les deux PDP.

80. Le député socialiste de Lorient L'Hévéder, pacifiste et paul-fauriste, siège aussi au Conseil national jusqu'en 1943. 


\section{RESUME}

Vue de l'extérieur, la Bretagne présente souvent l'image d'une région de droite alors que la réalité politique est plus complexe. Les années 1930 conduisent cependant les notables et les forces politiques de droite à s'organiser en partis modernes pour mieux canaliser le mécontentement des ruraux lors de la grande dépression. Ces partis de droite (Fédération républicaine-URD) se structurent autour de leurs parlementaires contre les gauches au début des années 1930 en incluant les centristes laïques (Alliance démocratique) et démocrates chrétiens (PDP). Il s'agit de ne pas être débordé par l'agitation des ligues faiblement implantées de la droite extrême (Jeunesses patriotes, Croix-de-Feu) et surtout par le dorgérisme que de nombreux notables accompagnent, puis par le PSF. Cette droite bretonne se durcit contre le Front populaire en s'appuyant souvent sur des hommes nouveaux. De 1936 à 1938, des reclassements s'opèrent dans une polarisation gauche-droite qui affaiblit les éléments centristes.

\section{ABSTRACT}

The notables and the rightist political forces in Brittany in the thirties

Seen from the outside, Brittany often shows the image of a rightist area, whereas the political reality is more complex. In the thirties however the notables and the political forces of the right system are meant to organise themselves in modem parties in order to channel better the discontent of the rural population during the great depression. These rightist parties (Fédération républicaine - URD) are structured around their deputies against the leftist parties in the early thirties including the lay centrists (Alliance démocratique) and the Chrétiens democrates (PDP). It's necessary not to let the agitation of the weakly rooted leagues of the extreme right (Jeunesses patriotes, Croix-de-Feu) and meanly the dorgerisme that is favoured by numerous notables, then the PSF, become out of hand. This breton right gets tough with the Front populaire often relying on the support of newcomers. From 1936 until 1938, reclassifyings are made in a right-left polarisation which weakens the centrist elements. 
